\title{
Nuevo enfoque en metodología de transferencia en los regadíos.
}

\author{
Jarén Morilla $C^{1}$, Salvatierra Bellido $B^{1}$, López Rodríguez $\mathbf{M}^{1}$
}

\begin{abstract}
Instituto de Investigación y Formación Agraria de Andalucía, Rancho de la Merced (IFAPA, sede Chipiona-Cádiz); cristina.jaren@juntadeandalucia.es, benito.salvatierra@juntadeandalucia.es, manuel.lopez.rodriguez@juntadeandalucia.es
\end{abstract}

Resumen: La transferencia de tecnología de forma amplia se suele enunciar como el proceso por el que se transfieren habilidades, conocimientos, formas de hacer, instrumentos... Con una conceptualización industrial, la transferencia de tecnología se dibuja como un proceso cuasi lineal desde los centros de conocimiento (universidades,...), donde algo que se descubre, que puede tener una utilidad, es introducido/vendido en un sector determinado. Muchas veces sin tener claro que esa nueva utilidad sea realmente la respuesta a una necesidad. El modelo simple sería "tengo un útil, ¿cómo vendo esto?".

Esta forma de realizar la transferencia ha hecho que, en muchos casos, el objeto de dicha transferencia no sea muy proclive a seguir las recomendaciones/transferencia que se les proponen. El sector agrario cada vez tiene menos peso en la economía y trabajo de los países desarrollados. En dicho sector, las innovaciones no aparecen con la celeridad que se produce en otros sectores, de ahí su desfase. Pero al mismo tiempo, cada vez más, juega un papel muy importante en las nuevas demandas de la sociedad como el medio ambiente y el cambio climático.

Los organismos de transferencia agro-alimentaria han realizado su labor con un modelo similar al de las industrias clásicas. En este trabajo se propone y se pone en práctica una metodología de transferencia que va más allá de las bondades del modelo de abajo a arriba donde el agricultor es el eje principal de la acción) utilizado en los últimos tiempos, se emplea además una visión desde lo general a lo concreto y se suman elementos de más horizontalidad analizando/integrando diversos aspectos que al final inciden en la rentabilidad y sostenibilidad de la explotación.

Para ello, se ha puesto el foco en una zona regable, el sector B-XII en Lebrija y se han unido cuatro proyectos que trataban partes aparentemente individuales para hacer un enfoque común de los mismos sin perder el trabajo de lo concreto pero desde una óptica que se inicia en lo general. Los proyectos han sido sobre riego, nitratos, suelos y teledetección. Al mismo tiempo, se han realizado convenios de colaboración con cooperativas, la comunidad de regantes y agricultores a los que se les ha realizado una monitorización de sus labores.

Este tipo de trabajo permite calar con mayor celeridad en los "modus operandi" de los técnicos y posteriormente de los agricultores con un mensaje claro donde el objetivo es mejorar la rentabilidad (entendida en un sentido amplio económica, social y ambiental) de la explotación.

Palabras clave: Transferencia; riego; horizontalidad; integración. 


\title{
Nuevo enfoque en metodología de transferencia en los regadíos.
}

\author{
Jarén Morilla $C^{1}$, Salvatierra Bellido $\mathbf{B}^{1}$, López Rodríguez $\mathbf{M}^{1}$
}

Instituto de Investigación y Formación Agraria de Andalucía, Rancho de la Merced (IFAPA, sede Chipiona-Cádiz); cristina.jaren@juntadeandalucia.es, benito.salvatierra@juntadeandalucia.es, manuel.lopez.rodriguez@juntadeandalucia.es

Abstract: The transfer of technology in a broad way is usually enunciated as the process by which skills, knowledge, ways of doing, instruments are transferred ... With an industrial conceptualization, technology transfer is drawn as a quasi-linear process from knowledge centers ( universities,...), where something that is discovered, that may have a utility, is introduced / sold in a certain sector. Many times without being clear that this new utility is really the answer to a need. The simple model would be "I have a tool, how do I sell this?".

This way of carrying out the transfer has meant that, in many cases, the object of said transfer is not very inclined to follow the recommendations / transfer that are proposed to them. The agricultural sector has less and less weight in the economy and work of developed countries. In this sector, innovations do not appear with the speed that occurs in other sectors, hence their lag. But at the same time, increasingly, it plays a very important role in the new demands of society such as the environment and climate change.

Agro-food transfer agencies have carried out their work with a model similar to that of classical industries. In this work, a transfer methodology is proposed and put into practice that goes beyond the benefits of the bottom-up model where the farmer is the main axis of action) used in recent times, a vision is also used from the general to the concrete and elements of more horizontality are added analyzing / integrating various aspects that ultimately affect the profitability and sustainability of the exploitation.

For this, the focus has been placed on an irrigable area, the B-XII sector in Lebrija and four projects that dealt with apparently individual parts have been joined to make a common approach to them without losing the work of the concrete but from a perspective that starts in general. The projects have been on irrigation, nitrates, soils and remote sensing. At the same time, collaboration agreements have been made with cooperatives, the community of irrigators and farmers who have been monitored for their work.

This type of work allows to penetrate more quickly the "modus operandi" of the technicians and later of the farmers with a clear message where the objective is to improve the profitability (understood in a broad economic, social and environmental sense) of the exploitation.

Keywords: Transferencia; riego; horizontalidad; integración. 


\section{Congreso Nacional de Riegos CARTAGENA 2021}

\section{Introducción.}

En el punto actual donde disponemos de mucha tecnología, la eficiencia en la aplicación de insumos, agua de riego (necesidades de la planta para su normal desarrollo/agua utilizada), fertilizantes y fitosanitarios es aún muy baja en término medio.

Los modelos de transferencia tradicionalmente se han desarrollado a partir de experiencias aisladas, en parcelas pequeñas o individuos no muy representativos de lo que se quería difundir (más si es una nueva forma de hacer). Normalmente, los individuos objeto tienen muy poca confianza, bien porque no se transmite adecuadamente o bien por la precariedad que genera la parcialidad de muchas experiencia.

Actualmente se disponen de herramientas que hacen posible un análisis global de amplias zonas que ayudan en la estrategia para introducir una tecnología.

En el B-XII con 14.643 has y 1.143 [1]parcelas se desarrolla una agricultura muy puntera donde se suceden a lo largo del año, cultivos hortícolas de consumo directo, tomate industrial, algodón, cereales,... con unos problemas de homogeneidad de producción, uso de insumos, cantidad de agua y de impacto ambiental, muy comunes todos en las zonas regables del Guadalquivir.

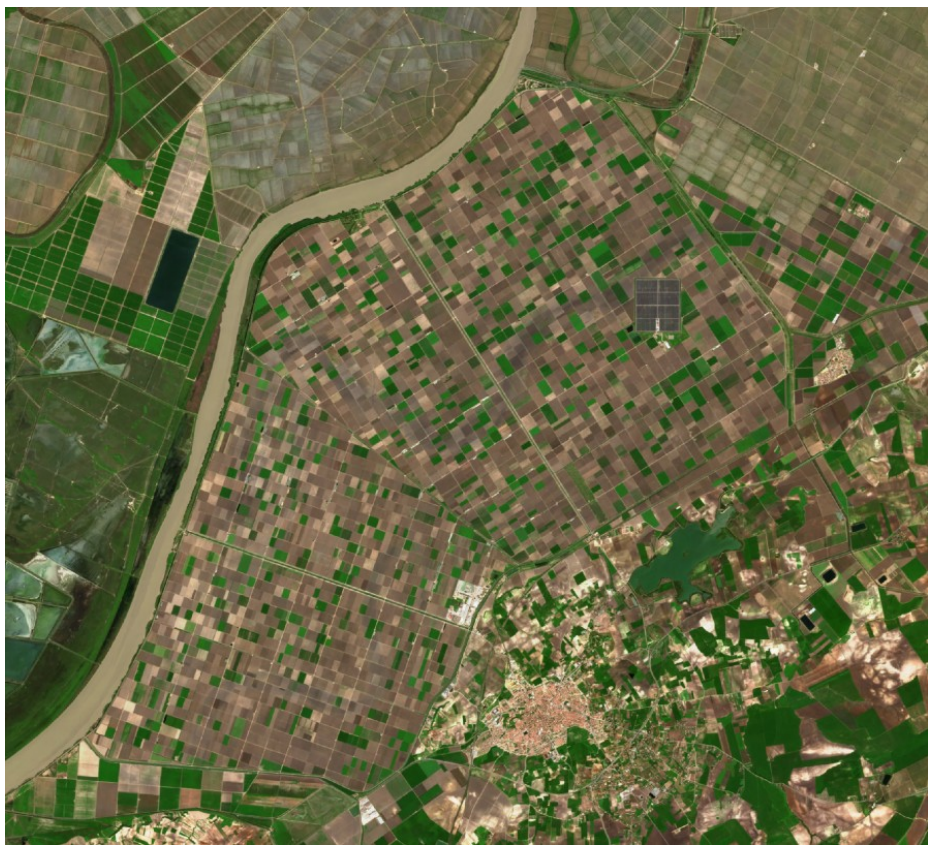

Figura 1. Imagen satélite de la zona regable sector B-XII.

Atendiendo a al Real Decreto 261/1996, de 16 febrero,[2] sobre protección de las aguas contra la contaminación producida por los nitratos procedentes de fuentes agrarias, esta zona, además sufrir episodios de restricciones de agua, en 2020, en dicho sector por pertenecer a unas de las zonas indicadas, se decide realizar un ensayo en una parcela de tomate de industria en el cual se establecen cuatro tratamientos experimentales [3]. Dichos tratamientos son el resultado de combinar 
un manejo sostenible cuyas pautas establecía el IFAPA y otro convencional bajo las indicaciones del propio agricultor coincidiendo en el manejo tradicional que el ejercía sobre sus parcelas.

De este dicho ensayo surgieron conclusiones muy interesantes que establecen el punto de partida del presente trabajo. Dichas conclusiones quedan relegadas a las características del ensayo en cuanto a superficie, dando lugar igualmente a la incógnita de la similitud de dichos resultados en condiciones más reales en cuanto a dicha superficie de cultivo se refiere.

Por todo ello, el objetivo pasó a ser el de realizar un diagnóstico de la zona y cambiar formas de operar con la fertirrigación para lograr mejoras económicas y ambientales en el desarrollo de la zona regable.

De esta forma, recogeríamos los diferentes escenarios en cuanto a riego y fertirrigación se refiere llevados a cabo en la zona para poder así extrapolar las conclusiones a escenarios reales de cultivo.

La metodología que se propone es fruto de aunar las herramientas y recursos que disponemos para poderlo fundir en una única visión generalizada de los cultivos en su escenario cotidiano.

En los últimos años, el equipo de trabajo del IFAPA Rancho de la Merced, ha estado realizando trabajos tendentes a mejorar la eficiencia del riego y de disminución del impacto del abonado en el medio, así como trabajos sobre el comportamiento de los suelos y más recientemente de teledetección (dron y satélites). A pesar de que los resultados obtenidos son muy prometedores y en algunos casos corroboran resultados ya muy antiguos, la realidad al analizar la zona elegida es que la eficiencia del riego es muy baja y el impacto en el uso de insumos es muy elevado en general.

Conscientes de esta realidad, en el último año se plantea una nueva metodología que pretende abordar el agrosistema del B-XII de forma global, tal cual es, y movilizando al mayor número de técnicos y agricultores que permitan la difusión de los resultados obtenidos con unas mayores posibilidades de éxitos y adelantándonos a un cambio en las formas de hacer que hagan del agrosistema B-XII un ejemplo de buen hacer en la agricultura sin por ello renunciar a la rentabilidad que como buenos agentes económicos los agricultores pretenden.

Así, el objetivo que planteamos con este nuevo enfoque es acelerar la transferencia de tecnología en el sector agrícola.

\section{Materiales y métodos.}

Para poder llevar a cabo el objetivo propuesto, el IFAPA Rancho de la Merced sede de Chipiona, ha hecho uso de herramientas de colaboración como son los convenios con empresas y los seminarios de discusión [4]para poder realizar la recopilación de información de primera mano como puede ser la de los propios técnicos encargados de las parcelas elegidas, que, a su vez, están en permanente contacto con los agricultores.

Dichas colaboraciones han tenido lugar mediante un convenio de colaboración con COALSA (agrupación de cooperativas algodoneras), Comunidad de Regantes Sector BXII y cooperativa Las Marismas de Lebrija S.C.A. Con esta última se ha realizado el seminario de discusión.

Además, se ha utilizado como material de apoyo el uso de programas de software libre como es Qgis donde se puede volcar la información recogida por imágenes satelitales, vuelos de dron y otras fuentes de información que se pueden obtener de manera gratuita y que complementan la visión 


\section{Congreso Nacional de Riegos CARTAGENA 2021}

global que queremos obtener de las parcelas. El uso de drones para el control de pequeñas parcelas demostrativas ofrece unas imágenes con una resolución óptima en comparación con las proporcionadas por los satélites que la captan a un tamaño de pixel más grande y por lo tanto la información que aportan es de menor exactitud.

Se decide trabajar con los cultivos de algodón y tomate de industria por tratarse de los más representativos de la zona.

La metodología llevada a cabo con todo lo mencionado anteriormente se detalla en el siguiente esquema:

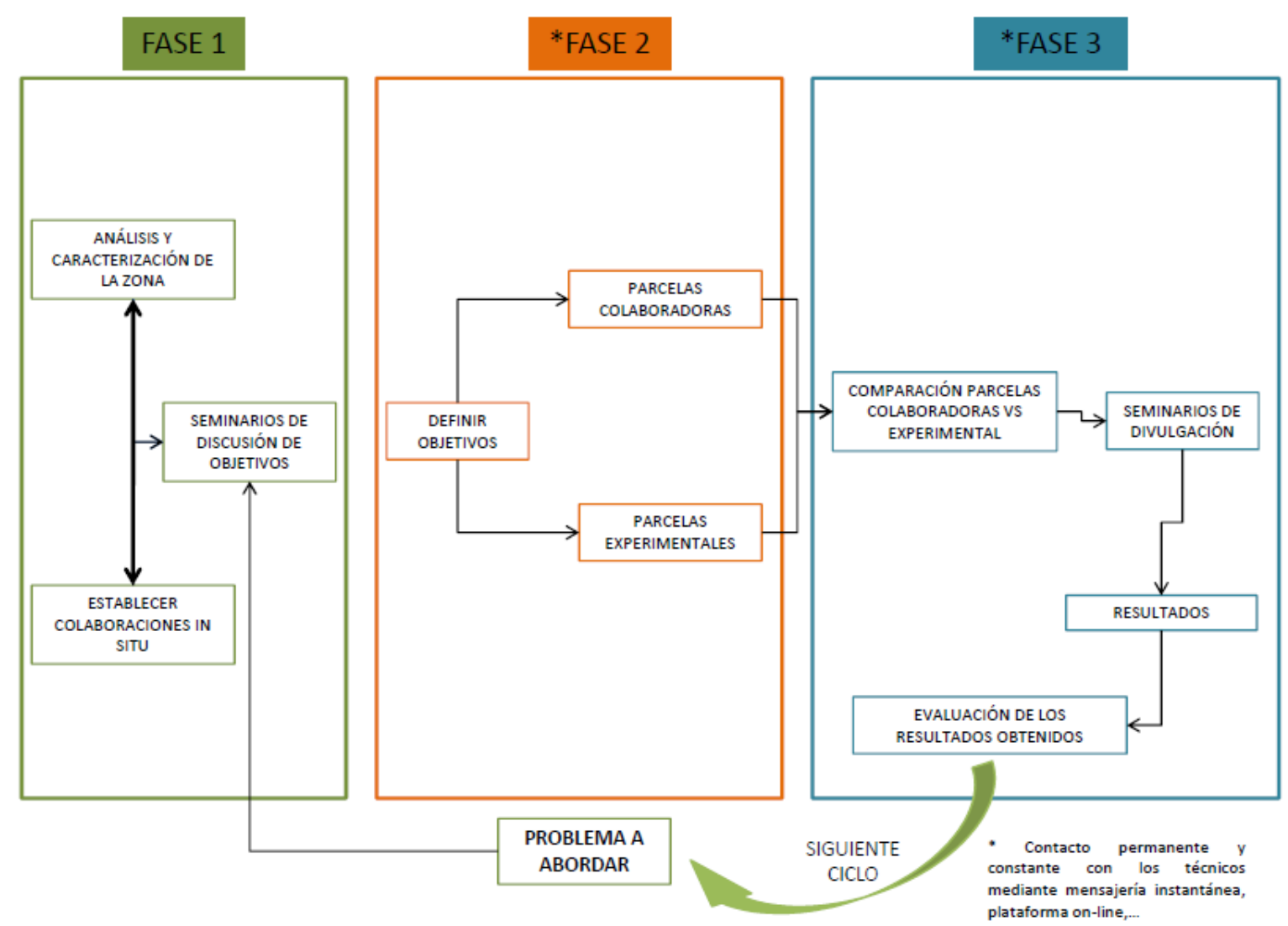

Figura 2. Esquema de la metodología empleada.

Se trata de reuniones mensuales donde se establece contacto directo con los técnicos que están a pie de campo de las parcelas colaboradoras elegidas en los convenios. En estos encuentros se revisa la información recopilada y se muestran observaciones que hasta la fecha se han ido realizando de manera general. También se resuelven dudas planteadas y se dan nociones acerca del manejo del riego y de la fertirrigación en base a la información recopilada.

Todo ello va apoyado en herramientas informáticas como es el uso de un espacio de trabajo online común, pero a la vez privado para cada técnico donde se puede incorporar la información requerida y realizar comentarios exclusivos y concretos para cada parcela. A su vez, se incorpora a 
cada carpeta una imagen por satélite donde se va detallando el índice de vigor de cada parcela solicitando por parte del técnico la descripción de la misma en caso de que se observen diferencias. También esta metodología se apoya en la realización de videoconferencias con los propios técnicos y el uso de mensajería instantánea para dar indicaciones de carácter general.

La duración de este seminario es la propia de los cultivos sobre los que se esté trabajando que en el caso que nos ocupa sería el de tomate de industria y algodón.

Una vez concluidos los cultivos se procede a una última reunión donde se pone en común las conclusiones obtenidas de manera general e incluso la posibilidad de plantear nuevas líneas de trabajo para el ciclo siguiente y que puede dar lugar a la realización de cambios importantes sobre el manejo de la fertirrigación y el riego en los cultivos estudiados.

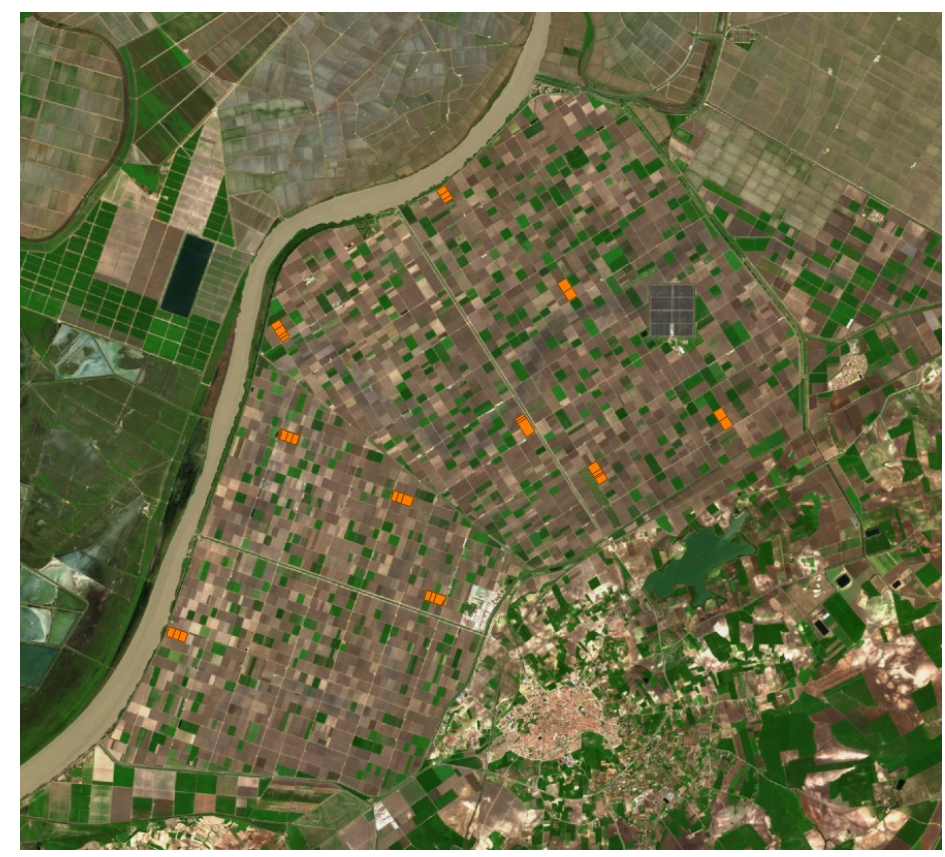

Figura 3. Ubicación de las parcelas colaboradoras de tomate de industria en el sector B- XII.

\section{Resultados y discusión.}

Como ya se ha comentado la zona de actuación pertenece a una de las zonas declaradas como vulnerables en la contaminación por nitratos y a su vez está sometida cada año a restricciones de agua de riego con toda la incertidumbre que ello conlleva a la hora de organizar los cultivos de la zona.

En base a estas dos premisas, eficiencia del agua de riego entendida como el agua de riego aprovechada realmente por la planta y el uso excesivo de insumos que incrementan los costes del cultivo y favorecen la contaminación del agrosistema, se decide actuar para transferir una metodología de aplicación de estos dos factores mediante el uso de prácticas más correctas de los mismos que ayuden a velar por el buen estado ambiental de la zona así como la rentabilidad de los cultivos.

Se definen dos líneas de trabajo complementarias que son el riego y la fertilización que se trabajan sobre una parcela experimental. En el caso del riego se establece la pauta de aportar la dosis de agua 
calculada expresamente en cada momento del ciclo del cultivo y por lo tanto aumentando la frecuencia del riego [5-7]. La fertilización se pauta en función de los requerimientos nutricionales de la planta a lo largo del cultivo a una dosis inferior a la propuesta por la producción integrada [8] donde además, se elimina el aporte de abonado de fondo y se establece la frecuencia de fertirrigación manteniendo una proporción 10-80-10 con respecto al riego, donde el aporte de nutrientes se realiza en cada riego y a lo largo del tiempo que dura el mismo, salvo un tiempo mínimo inicial para la estabilización del sistema de riego y otro final para la limpieza de dicho sistema.

En las parcelas colaboradoras se establecen los criterios de similitud de superficie cultivada, fecha de plantación, cultivar, existencia de drenes,... con respecto a la parcela experimental pero el manejo es el propio del agricultor.

Mediante el empleo de sistemas de información geográfica y teledetección se comparan los resultados obtenidos de la parcela experimental con el resto de parcelas colaboradoras obteniendose así una serie de conclusiones que se trasladan los técnicos responsables de dichas parcelas.

La siguiente imagen muestra los resultados obtenidos concretamente en las parcelas de tomate de industria estudiadas.

Los resultados esperados de la aplicación de esta metodología de trabajo son básicamente, pero no por ello menos importante, el contabilizar el número de agricultores deciden incorporar esas buenas prácticas en riego y fertilización al comprobar en parcelas similares a las suyas que no sólo la producción se mantiene o incluso es mayor, sino que además, el gasto en insumos es menor por lo que al final, la rentabilidad aumenta sin sacrificar el coste ambiental, logrando de esta forma que la zona cambie en un periodo de varios años tanto el perfil del manejo del riego como el de abonado lo que a su vez se traducirá en una mejora ambiental de la zona pero no por ello una disminución de la rentabilidad.

La figura siguiente muestra los resultados de producción en tomate de industria recogidos a través de la información facilitada por los técnicos colaboradores.

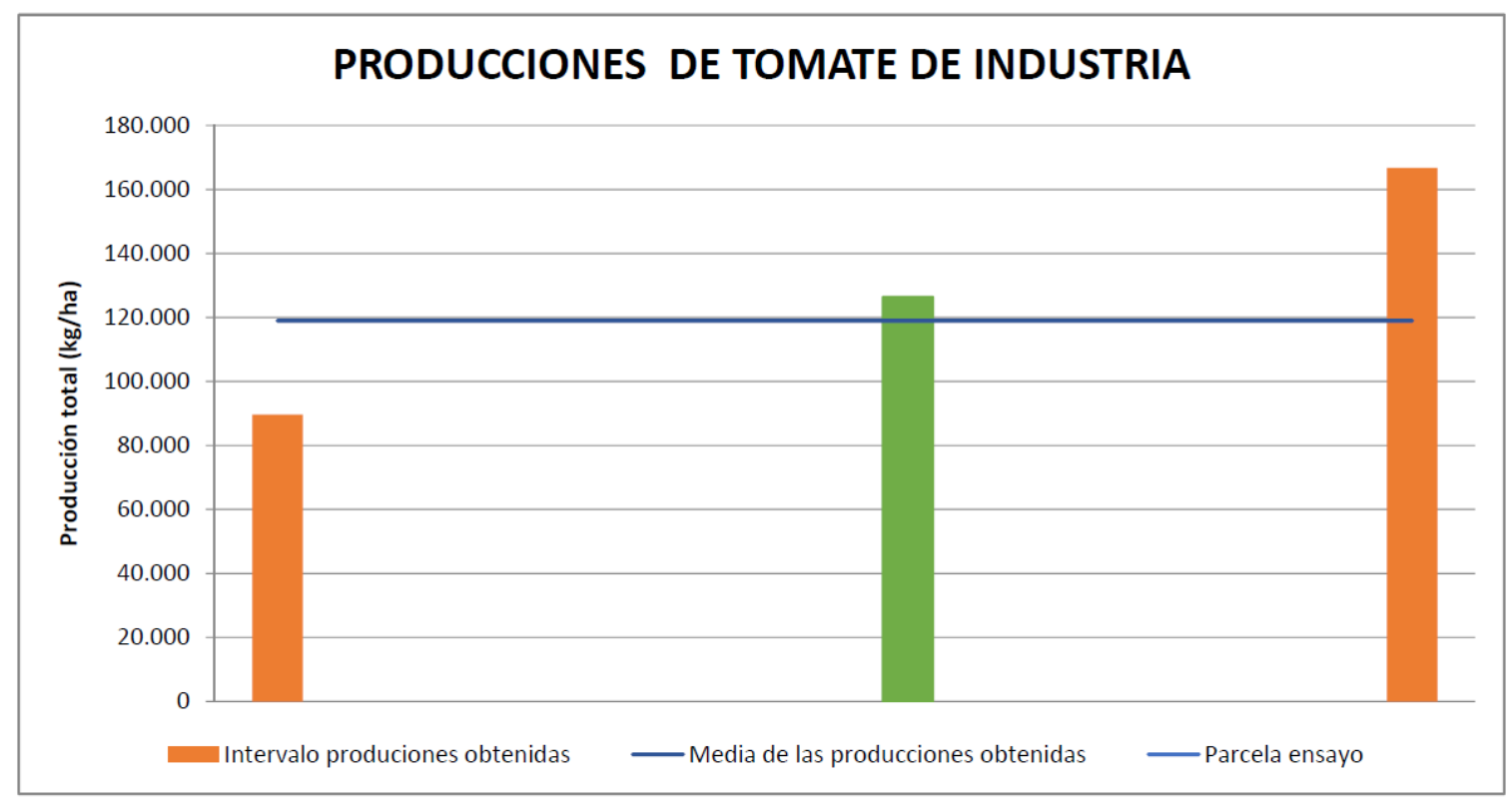

Figura 4. Producciones obtenidas en las parcelas colaboradoras con tomate de industria. 
La producciones obtenidas en las parcelas colaboradoras de tomate de industria están comprendidas entre los 85.000 y los $165.000 \mathrm{~kg} / \mathrm{ha}$, quedando la establecida como parcela experimental con una producción que supera a la homóloga manejada por propio agricultor de la parcela, en una producción en un 30\%. También se puede observar como la producción de la parcela manejada bajo los criterios del IFAPA queda por encima de la media obtenida en la zona.

\section{Conclusiones}

El hecho de actuar sobre parcelas grandes de extensión y características similares a las de la zona, así como la integración de las técnicas, hace que el agricultor perciba mayor realismo en los trabajos y esté más dispuesto a colaborar en la adopción de nuevas formas de hacer sobre los cultivos. Además, al involucrarlos en la propia metodología [9] ven de primera mano la veracidad de los resultados animándose a adoptar la propuesta como forma de hacer.

Esta metodología requiere que se repita de manera cíclica para poder ir identificando y ajustando problemáticas que existan o que puedan ir surgiendo.

El indicador en el éxito de la transferencia deberá ser el número de agentes que hacen suya la técnica propuesta en un área determinada. Lo cual requiere de unos plazos de tiempo mayores a los de una sola campaña.

\section{Referencias}

1 Comunidad de Regantes del Sector BXII del bajo Guadalquivir. Septiembre, 2021. http://www.crsectorb12.es

España. Real Decreto 261/1996, de 16 febrero, sobre protección de las aguas contra la contaminación producida por los nitratos procedentes de fuentes agrarias. Boletín Oficial del Estado, de 11 marzo de 1996, núm. 61.

Salvatierra, B., Jarén, C., López, M., Vanderlinden, K. (2021). Optimización del riego y la fertirrigación en cultivo del tomate de industria. Agricultura,1048 (marzo), 50-56. http://www.revistaagricultura.com.

Salvatierra, B. El seminario continuo de asesoramiento al regante como formato de formación para la transferencia en riegos. Instituto de Investigación y Formación Agraria, Pesquera, Alimentaria y de la Producción Ecológica, IFAPA, 2010.

Organización de las Naciones Unidas para la Alimentación y la Agricultura, 2006. Evapotranspiración del cultivo. Guía para la determinación de los requerimientos de agua de los cultivos. Estudios de Riego y Drenaje 56.

Hanson, B. y D. May. 2006. Coeficientes de cultivo para tomate procesado con riego por goteo. Gestión agrícola 81: $381-399$.

Campillo, C. et al. Manual práctico de riego tomate de industria. Centro de Invetigaciones Científicas y Tecnológicas de Estremadura, CICYTEX, 2015.

Orden de 2 de octubre de 2008. Reglamento Específico de Produccción Integrada de Tomate para Transformación Industrial. Boletín Oficial de la Junta de Andalucía, 203, de 10 de octubre de 2008, 13-24.

Kamruzzaman, M.; Daniell, K.A. ; Chowdhury, A.;Crimp, S.;James, H., 2020. How can agricultural extension and rural advisory services support innovation to adapt to climate change in the agriculture sector?. Advancements in Agricultural Development, 1 (1), 48-62. 